\title{
Harnessing CRISPR/Cas9 Technology in Cardiovascular Disease
}

Hamzeh Rezaei, Saedeh Khadempar, Najmeh Farahani, Elaheh Zadeh Hosseingholi, Seyed Mohammad Gheibi Hayat, Thozhukat Sathyapalan, Amir Hossein Sahebkar

Running title: CRISPR/Cas9 and Cardiovascular Disease

\begin{abstract}
The CRISPR/Cas9 system is a precisely targeted bacterial defense system, used to control invading viruses. This technology has many potential applications including genetic changes in somatic and germ cells and the creation of knockout animals. Compared to other genome editing techniques such as zinc-finger nucleases and transcription activator-like effector nucleases (TALENS), the CRISPR/Cas9 system is much easier and more efficient. Most importantly, the multifunctional capacity of this technology allows simultaneous editing of several genes. The CRISPR/Cas9 system also potentially has the ability to prevent and treat human diseases. The present article addresses some key points related to the use of the CRISPR/Cas9 system as a powerful tool in cardiovascular research and as a new strategy for the treatment of cardiovascular disease (CVD).
\end{abstract}

Key words: CRISPR/Cas9; Genome editing; Cardiovascular disease 


\section{Introduction}

The drive towards personalized medicine has gained momentum among researchers in recent years. Scientists have succeeded in the in vitro treatment of cancer, congenital malformations, bacterial and viral infections, and the production of genetically modified food with the aid of the CRISPR (Clustered Regularly Interspaced Short Palindromic Repeats) system. The accumulation of individual characteristics, gleaned from genomics, proteomics and phenotypic parameters has not only directed research towards health and disease, but has also led to alterations in and optimization of available methods for calculating risk likelihood, diagnostic tests and therapeutic strategies (1).

In 1987, a group in Japan described the CRISPR system in Escherichia coli as 29-nucleotide repeats separated by 32 nucleotides as spacers, which protects bacteria and archaea against bacteriophage and plasmid invasion. These defense systems rely on a small RNA that detects specific sequences and extinguishes external nucleic acids (2). Indeed, the CRISPR system is a kind of safety system that lies in the genetic code of our cells. The CRISPR regions are nucleotide repeats separated by unique spacers. The structure of these DNA sequences is complementary for the genome of various viruses (bacteriophages) that attack bacteria. Later came the discovery of Cas proteins possessing the ability to cleave DNA strands into two. The Cas proteins form a complex with RNA obtained after transcribing the spacer sequence; they search inside the cell to find the DNA fragments of the invasive viruses with their complementary sequences in order to eliminate them using the Cas enzyme (3). In principle, viral DNA fragments, by being stored in the chromosome and creating memory for the bacterial immune system, are preparing the virus for subsequent attacks. The human immune system works similarly, but in a more advanced manner. To date, six different CRISPR systems have 
been discovered, initially three types from I to III, followed by IV to VI. The characteristics of each system, such as the mechanism of transmission, expression and change to identify and create a gap in the target DNA, are different according to their individual mechanisms during the process of immunogenicity. In general, researchers only use the bacterial type II CRISPR/CAS9 system, which includes guide RNA (gRNA), as the best option for generating changes in the genome as well as genetic engineering (4).

The type II CRISPR/Cas9 system exploits a single Cas9 nuclease. This enzyme acts in coordination with two gRNAs of crRNA (CRISPR RNA) and tracRNA (transacting CRISPR RNA). To optimize the usage of this system, researchers apply a joining loop to generate a dualtrac RNA called a single guide RNA (sgRNA), which, together with Cas9 endonuclease, creates a specific gap in the target sequence. A conserved 2-5 base pair DNA sequence called protospacer adjacent motif (PAM) reading in the opposite direction to the RNA-DNA hybrid is essential for identifying the target DNA sequence by Cas9.

Upon detecting the target sequence, a double-strand cleavage occurs by the two Cas9 domains. These domains consist of HNH (to cleave crRNA complementary strand) and RuvC-like (to cleave the template strand). By using this mechanism, the nuclease designed with sgRNA is capable of creating a genomic DNA cleavage in a specific site, resulting in an accurate and definitive change in the genome. Modification of a small number of gRNA sequence bases, which are complementary to the target sequence, together with the Cas9 protein, allows new targets to be easily defined. The capacity to induce multiplex double-strand cleavages in a genome due to the expression of different types of RNA in the system is among other capabilities of the Cas9 protein (5). Following the DNA double-strand cleavage, the damage is corrected by 
one or two major repair paths, Homology Directed Repair (HDR) and NHEJ (Non-Homologous End Joining).

The NHEJ is an error-prone method in which double-strand cleavage is repaired via short deletions and insertions; therefore, researchers often use this system to induce mutation and deactivation of the gene. In contrast, HDR is a precise method as the repair mechanism is based on the use of a template strand; hence, this route is mainly applied to delete or duplicate precisely the fragments of the target genome, or to precisely insert an external gene into a predetermined site in the genome (6). To sum up, pros and cons of these methods can be stated as follows: NHEJ-break ends can be ligated without a homologous template, whereas HDR-breaks requires a template to guide repair. NHEJ is a very efficient repair mechanism that is most active in the cell. It is also susceptible to frequent mutation errors due to nucleotide insertions and deletions (indels). HDR is considered the dominant mechanism for precise DSB repair, but suffers from low efficiency as it requires higher sequence similarity between the severed and intact donor strands of DNA. There are fewer errors or chances of mutations if the DNA template used during repair is identical to the original undamaged DNA sequence To make changes in a gene using CRISPR , the population of transfected cells will contain a mixture of NHEJ-repaired and HDRrepaired alleles. HDR-edited DNA is much more attractive to guarantee controlled modifications

\section{Ex vivo and in vivo studies in animal models:}

Today, the application of the CRISPR/Cas9 method has expanded into a variety of areas, including agriculture, husbandry, disease patterns and targeted therapies. This section emphasizes the therapeutic aspects for diseases with a genetic basis, especially monogenic disorders (9). In gene therapy, a gene with pathologic properties can be modified by both ex vivo 
and in vivo approaches. In the ex vivo approach, the targeted cell population is removed from the patient's body, and the designed nucleases are used to induce the desired changes and then returned to the patient by grafting. Avoiding issues such as transplant rejection and immune responses are important benefits of this method. Using in vivo gene therapy, the genomic modifying factors, such as programmatic nucleases and patterns, are put directly into the patient (10).

\section{Gene therapy for genetic diseases:}

Gene therapy refers to manipulating sequences and stimulating deletion mutations to provide protective function in the host. The optimal diseases to target with gene therapy are monogenic disorders. The acquisition of only one copy of an impaired gene can be offset by one normal gene and thereby prevent disease development. Polygenic disorders involve multiple variations in the genome simultaneously, creating a major challenge in the treatment of these diseases.

Targeted alterations of genes, both in somatic cells and in germ cell lines, have been reported in animal models. The treatment of a particular disease clearly requires knowledge of its genetic basis. Molecular scissors can be used to create multiple changes in the genome and repress disease development. Access to genetic information on complex diseases has enabled genome editing technology (GET) to offer opportunity for treatment of complex multifactorial illnesses, such as cardiovascular disease (11).

In a recent study, a mutation in the MYBPC3 gene was targeted using the CRISPR-Cas genomic editing technique to correct this mutation in a human embryo with hypertrophic cardiomyopathy, a condition that would eventually cause heart failure. Interestingly, the paternal mutated gene, when restricted by CRISPR-Cas9, is more efficiently replaced by the maternal gene rather than 
by synthesized DNA. Human embryonic manipulation was much more efficient (72.2\%) than that of iPSC stem cells (17.61\%-27.9\%). This suggests that the human embryo utilizes the DNA repair mechanism differently from somatic and stem cells. Using Cas9 protein injection prevented any off-target effects (12).

\section{Cardiovascular disease}

Cardiovascular disease (CVD) is the leading cause of mortality globally (13). CVD is widely associated with both inherited and non-inherited diseases. While personalized medicine has become a goal, and genome sequencing data are increasingly available from patients, the experimental and functional studies enabling understanding of how genetic differences are involved in promoting disease are lacking. Recent GETs have enabled studies of biological function of genes in both cell systems and animal models (14).

In this review, we highlight the latest technologies for genome editing, describe their use in different in vitro and ex vivo systems, and focus on how technological advances in genome editing may lead to deeper understanding of, and treatment for, CVD (15).

\section{Therapeutic potential of the CRISPR/Cas9 system for the treatment of CVD}

The CRISPR/Cas9 system can be used for therapeutic purposes, not only by the correction of disease-related mutations, but also through introduction of protective mutations and targeting of viral genomes (16). Recently, Limpitikul et al. edited a mutation in the Calmodulin 2 (CALM2) gene in the iPSC-CM, thereby preventing cardiac arrthymias due to long QT syndrome (LQTS) (17). Calmodulin-related diseases (Calmodulinopathies) associated with LQTS occur due to a mutation in one of the CALM1, CALM2, and CALM3 genes. In this case, the proband under the 
influence of the malignant calmodulinopathic LQTS is located on only one of the six coded alleles of calmodulin. Here, a CRISPR intervention strategy (CRISPRi) was used to select only the mutated allele. The CRISPRi technology uses the dCas9 protein to regulate gene expression at the transcriptional level. The dCas9-sgRNA complex binds to the targeted gene in the coded or coding tail, inhibits the polymerization activity of RNA and prevents the onset of transcription or elongation (18). With successful inhibition of the mutated allele, the authors have proposed that this method can be applied, not only to other calmodulinopathies such as catecholaminergic polymorphic ventricular tachycardia (CPVT), but also to a variety of cardiac and non-cardiac diseases characterized by transcription of a damaged gene (19). Although attractive as a strategy, the efficacy of genomic editing methods for treatment depends upon the development of a successful strategy for the CRISPR/Cas9 system in the patients. This can be obtained ex vivo using a patient-to-patient strategy via editing and then transplanting back into the patient, or by directly delivering the CRISPR / Cas9 system to the desired site in the specific tissue (Fig. 1 (a) and 1 (b)) (19).

The CRISPR/CAS9 system can target many tissues and organs; however, it is difficult to effectively limit it to only a particular targeted tissue. The majority of ex vivo studies insert the CRISPR/Cas9 components using adeno-associated viral vectors (AAVs), but AAV tissue tropism is limited to certain organs (20).

\section{In vitro methods for CRISPR/Cas9}

Although in vitro models, such as modified cell culture, are widely used because they are relatively easy to maintain and manage including simplicity, species specificity, convenience and automation. It is true that these can not reflect the complexity of the various cells in 
physiological processes, but the benefits mentioned above are valuable in that they are still exciting to use.(15). One attractive in vitro system is human pluripotent stem cell lines (hPSC lines). These patient-derived cells are generated from induced pluripotent stem cells (iPSC), and can be reprogrammed and then differentiated into a variety of cells for functional analysis. However, comparing iPSC lines from different individuals can be confusing because of differing epigenetics and differing capacities to differentiate into the type of desired cell. The creation of homogeneous and isogenic cell lines using genome editing to introduce specific changes in the field (reprogramming and differentiation) of interest can circumvent this issue, providing a more reproducible model of genetic changes. The production of isogenic hPSC lines using Zinc Finger Nucleases (ZFNs are engineered DNA binding proteins that can be designed to bind to a wide variety of DNA sequences and function by introducing a double stranded break at a specified location in the genome)(21), Transcription activator-like effector nuclease (TALEN technology leverages artificial restriction enzymes generated by fusing a TAL effector DNAbinding domain to a DNA cleavage domain) (22) or CRISPR/Cas9 has increased knowledge about cardiomyopathy, including Barth syndrome (a mitochondrial dysfunction disorder caused by mutations in the tafazzin (TAZ) gene). Gene replacement and genome editing have shown that a TAZ mutation is both necessary and sufficient for the manifestation of these phenotypes, as sarcomere assembly and abnormalities of myocardial contractility have occurred despite total ATP levels of the cell falling within normal limits (23).

According to published reports, TALENs uses 15 genes in somatic cell cultures or humanderived stem cells to produce rapid and efficient mutated alleles (24). Other uses are in lipid disorders (25), electrophysiological disorders (26), and valvular heart disease (27). Zhang et al. reported that CRISPR/CAS9 ablation of individual miRNAs from a miRNA family disclosed 
their individual efficacies in regulating cardiac differentiation. During differentiation of embryonic stem cells, individual miRNAs from the miRNA17 family (composed of 14 miRNAs) do not share the same function, even though they have the same seed sequence. The CRISPR/CAS9 technology is not only enlightening in terms of individual miRNA function, but also adds advanced molecular biology approaches to classical cell biology approaches such as gene rescue. Findings indicate that miRNA106a, and to a lesser extent miR17 and 93, can target the cardiac suppressor gene Fog2, which specifically suppresses Gata-4 and Coup-TF2. However, when each miRNA was individually knocked out, targeting efficacies for Fog2 differed, resulting in varying degrees of cardiac differentiation (28).

CRISPR/Cas9 was used to edit DMD mutations by deleting exons via exon skipping. In proofof-concept investigations, myoediting was performed in human-derived iPSCs from several patients with large deletions, point mutations or duplications within the DMD gene, and to efficiently engineer dystrophin protein expression in derivative cardiomyocytes. In 3D engineered heart muscle (EHM), myoediting of DMD mutations altered dystrophin expression and the corresponding mechanical force of contraction. Correcting only a subset of cardiomyocytes (30\% to 50\%) was adequate to edit the mutant EHM phenotype to levels close to normal. The authors concluded that the rejection of conserved RNA splicing acceptor/donor sites and the guidance of the splicing machinery to skip mutant or out-of-frame exons through myoediting allowed correction of DMD-related cardiac disorders (29).

Modeling disease using hPSCs was less than optimal because the influence of individual genetic variation on cell phenotype can be greater than from the pathogenic mutation; gene editing using Cas9/CRISPR solves this issue. A simplified PiggyBac (PB) strategy is a highly efficient transposon originally isolated from Trichoplusia ni,(30) like other transposons, has two 
components, a transposon and a transposase. The precise 'cut and paste' transposition mechanism makes PB a powerful tool for mutagenesis and transgenic manipulation. PB can accommodate relatively large DNA fragments without compromising transposition efficiency, exhibits significantly higher transposition efficiency in mammalian cells and multiple copies of PB can incorporate into the host genome providing for the possibility of multiple simultaneous manipulations of the host genome (31) ) shortened the hPSC correction by up to two weeks and needed only a single round of clonal expansion and genotyping rather than two rounds, delivering identical efficiencies when compared to the longer conventional process. A successful outcome was achieved across four heart-related sites (ADRB2, GRK5, RYR2, and ACTC1) with genomic cleavage and efficacy correction have been shown to be $8 \%-93 \%$ and $67 \%-8 \%$ respectively, which include mono and / or biallelic events. Differentiation into high-purity cardiomyocytes conserved pluripotency (CMs; 88\%-99\%). Using GRK5 isogenic lines as an exemplar, chronic stimulation with the $\beta$ adrenoceptor agonist isoprenaline decreased beat rate in hPSC-CMs expressing GRK5-Q41 but not GRK5-L41; this was reversed by the $\beta$-blocker propranolol. This footprint-free shortened approach will prove useful for mechanistic studies (32).

Following is an example of CRISPR/Cas9 application in modifying cholesterol levels, to better understand this method. In a study conducted by Hai, quantitative trait loci were mapped for the concentrations of free cholesterol and esterified cholesterol and the esterified/free cholesterol ratio was measured in acetylated bone marrow-derived macrophages (BMDMs) loaded by low-density lipoprotein (LDL), derived from an $A K R \times D B A / 2$ strain intercross. The prioritization of the studied loci suggested the Soat1 (sterol O-acyltransferase 1) gene-containing locus on distal chromosome 1, which encodes 
Acyl-coenzyme A:cholesterol acyltransferase 1 (ACAT1) and causes the esterification of the free cholesterol. The deletion of exon 2 was found in the parental AKR strain in Soat1, resulting in curtailment of 33 amino acids in the N-terminal end of ACAT1. The deletion of Soat1 exon 2 in the AKR strain was replicated by CRISPR/Cas9 gene edition strategy for DBA/2 embryonic stem (DBA/2 ES) cells, without change in the remainder of genome when leaving. The results showed the differentiation of DBA/2 ES cells and stem cells heterozygous/ homozygous for the Soat1 exon 2 deletion into macrophages, which were loaded with acetylated LDL. Less free cholesterol and higher esterified cholesterol were accumulated in the macrophages derived from DBA/2 ES cell compared to stem cells heterozygous/homozygous for the Soat1 exon 2 deletion (33).

\section{In vivo methods for CRISPR/Cas9}

In vivo genome editing requires the correction of various diseases in the primary cells in situ. A smaller cas9 protein from Staphylococcus aureus has recently been described that makes it possible to release CRISPR/Cas9 using adeno-associated virus vector (34). Proof-of-concept studies on preclinical mice have been carried out using genome editing. Using CRISPR/Cas9 by NHEJ, faulty exon can be cleaved or repaired and remove the dystrophin gene leading to Duchenne muscular dystrophy (DMD), resulting in significant improvement in muscle function (35).

In vivo therapeutic genome editing in the cardiovascular system faces several major barriers, however, catastrophic consequences may occur in rare cases, despite improvement in predictor algorithms. First, NHEJ correction mechanisms may lead to cellular dysfunction or tumor formation. Second, induction of double-strand cleavage of a healthy allele involved in regulating 
heterozygote mutations may lead to deterioration of the phenotype. Third, cellular correction mechanisms depend upon the stage of the cell cycle; while NHEJ can lead to DNA editing during G1, S, and G2 phases, normal HR is limited to the late S and G2 phases when sister chromatids are present (36). Since most cardiomyocytes in the postnatal heart are post-mitotic, new research seeks to apply HR correction to post-mitotic cells. However, NHEJ mediated exon skipping may be technically feasible especially for mutations in titin (TTN) and myosin binding protein C3 (MYBPC3) (37). Fourth, unlike skeletal muscle satellite cells that have a huge regenerative potential, the inherent regenerative capacity of an adult heart is highly limited. Therefore, a substantial number of cardiomyocytes must be successfully edited to induce improvements in cardiac function.

These physiological and technical limitations currently present, important challenges for in vivo genome editing for the treatment of cardiomyopathies (34). Beside cardiomyocytes, endothelial cells and smooth muscle cells might also be useful targets for in vivo genome editing in the treatment of CVD. One possible scenario is the correction of validated genomic variants in endothelial cells, as noted in genome-related studies (38-39). Different disease-causing or disease-modifying factors will likely be discovered due to more readily available whole exome and whole genome sequencing. However, not all of these factors are present in genes expressed specifically in endothelial or smooth muscle cells, making cell-specific delivery of CRISPR/Cas9 difficult for clinical applications. In this regard, CRISPR/Cas9 technology shows significant improvement over the limitations of conventional procedures (40-41).

Zebrafish have gained in popularity over mice as a developmental model of heart disease because of relative ease of performing genetic analysis. The delivery of adequate oxygen during the first week of development is not a limitation in zebrafish as it is in other models, therefore 
the zebrafish model allows the study of cardiovascular developmental abnormalities that would result in fetal death in other organisms. The zebrafish heart has great regeneration potential that is not found in other vertebrates such as rodents (42). Several CRISPR/Cas9-based systems for genome editing have been reported in zebrafish (43). Recently introduced is a tool to produce a gene-specific tissue by injection of gRNA and Cas9 mRNA into a single stage of the embryo cell. As previously described in Drosophila, this offers the possibility of controlling the disorder of a particular gene in the cardiomyocytes and markedly improves the ability to test loss of function in zebrafish, which could be used not only to delineate embryonic heart phenotypes but also to model adult heart disease (44).

The CRISPR/Cas9 system, which is directly applicable to embryos, has become a popular tool for research in rodent models. Older methods needed to induce homologous recombination in mouse embryonic stem cells, the selection of mutant ESCs through antibiotic resistance, the elimination of false antibiotics, and ESC injection into blastocyst-receiving mice (45). In contrast, the CRISPR/Cas9-based technology provides the possibility of producing mutated mice in only one step, including simultaneous injection into Cas9 mRNA sprouts, different sgRNA and DNA donors (46).

The CRISPR/Cas9 system can be used for in vivo gene editing in somatic cells. Suppression of the PCSK9 gene using the CRISPR/Cas9 tool resulted in lowered blood cholesterol levels and reduced coronary heart disease (CHD) risk (47). Chadwick et al. reported significantly reduced blood lipid levels after in vivo CRISPR-based genome editing in mice where functional mutations in Angptl3 encoding a lipoprotein lipase inhibitor were introduced. The treatment in wild type and $\mathrm{Ldlr}^{-/-}$mice was effective and had the same effect as Pcsk9 genome editing without causing unpredictable mutations (48). Regarding the comparison of CRISPR method with 
other therapies such as monoclonal antibodies alirocumab and evolocumab, it is important to acknowledge that these drugs received approval from the FDA in 2015 and long term safety is not yet established. An additional major concern is the high cost of PCSK9 inhibitors and their effect on healthcare costs as well as health insurance premiums (49).

\section{Conclusion}

Since its identification and development, the CRISPR/Cas9 system has been shown to be a powerful tool creation of new cell and animal models for the study of pathophysiological mechanisms in a number of diseases. The therapeutic potential of CRISPR/Cas9 for CVD is a challenge due to technical and biological barriers, especially the postmitotic nature of cardiomyocytes. Cardiac fibroblasts, endothelial cells and smooth muscle cells are the main cell targets using this technique. The development of advanced tools is needed to meet the goal of routine use of this technique in CVD therapy.

Conflict of interests: None. 


\section{Figure legends}

Figure 1. Functional structure of gRNA/Cas9 Complex

Figure 2. The CRISPR / Cas9 genome editing system has two components: a) endonuclease b) RNA short sequence guide. The last 20 bp of the gRNA are used as an Identifier of DNA sequencing and lead Endonuclease Cas9 to the target sequence. Then, Cas9 cuts DNA in the 3-4 bp upstream of the PAM sequence. Subsequently, DSB will be restored by NHEJ or HDR.

Figure 3. Diagram of the process that occurs within the CRISPR-Cas9 gene editing complex. In this figure in vivo and ex vivo gene therapy is explained diagrammatically. In this mode of gene therapy, genes are transferred to the cells grown in culture, and the transformed cells are selected, multiplied and then introduced into the patient.

Figure 4. Application of CRISPR in the treatment of cardiovascular diseases. The somatic cells extracted from the patient are induced to IPS by the reprogramming process, then the genetic modification with CRISPR is performed on them. The differentiation process is then instituted on the modified cells and eventually transmitted to the patient

Figure 5. Application of Genomic Editing for iPS( Cells). The creation of normal cells and targeted mutations cells using CRISPR.

Figure 6. Evaluation of the effectiveness of gRNA to correct various types of DMD mutations in iPSC cells using exon skipping in the form of myoediting.

Figure 7. Schematic to demonstrate the various ways to inhibit ANGPLT3 function. These include: a) Inhibiting transcripts using Antisense Oligonucleotide b) blocking antibodies c) CRISPR genomic editing with the aim of eliminating functional mutations, ultimately reducing levels of lipids. 
Table 1. Advantages and disadvantages of the CRISPR / Cas9 system (19, 50-51).

\begin{tabular}{|ll|ll|}
\hline \multicolumn{2}{|l|}{ Advantages } & \multicolumn{2}{|l|}{ Disadvantages } \\
\hline 1. & Site-specific gene targeting & 1. & Requirement of a PAM sequence in the target DNA and a "seed” region in the crRNA \\
2. & Effective, permanent mutations & 2. & Binding efficiency impacted by chromatin accessibility \\
3. & Screening DNA noncoding regions & 3. & Varying degrees of specificity \\
4. & Can induce insertions or deletions & 4. & Toxicity \\
5. & At gene loci or chromosomally & 5. & Off-target effects \\
6. & Heritable mutations & 6. & Certain sites cannot be mutagenized \\
7. & Generation of mutant lines & 7. & Mosaicism \\
8. & Can target multiple genes at once & 8. & Multiple alleles \\
9. & Fast and easy-to-use technique & 9. & Ethical issues \\
10. & Short sgRNAs & & \\
\hline
\end{tabular}




\section{References}

1. Jameson JL, Longo DL. Precision medicine-personalized, problematic, and promising. Obstetrical \& Gynecological Survey. 2015;70(10):612-4.

2. Ishino Y, Shinagawa H, Makino K, Amemura M, Nakata A. Nucleotide sequence of the iap gene, responsible for alkaline phosphatase isozyme conversion in Escherichia coli, and identification of the gene product. Journal of bacteriology. 1987;169(12):5429-33.

3. Hale CR, Zhao P, Olson S, Duff MO, Graveley BR, Wells L, et al. RNAguided RNA cleavage by a CRISPR RNA-Cas protein complex. Cell. 2009;139(5):945-56.

4. Charpentier E, Richter $\mathrm{H}$, van der Oost J, White MF. Biogenesis pathways of RNA guides in archaeal and bacterial CRISPR-Cas adaptive immunity. FEMS microbiology reviews. 2015;39(3):428-41.

5. Cong L, Ran FA, Cox D, Lin S, Barretto R, Habib N, et al. Multiplex genome engineering using CRISPR/Cas systems. Science. 2013:1231143.

6. Maruyama T, Dougan SK, Truttmann MC, Bilate AM, Ingram JR, Ploegh $\mathrm{HL}$. Increasing the efficiency of precise genome editing with CRISPR-Cas9 by inhibition of nonhomologous end joining. Nature biotechnology. 2015;33(5):538.

7. Ghezraoui H, Piganeau M, Renouf B, Renaud JB, Sallmyr A, Ruis B, et al. Chromosomal translocations in human cells are generated by canonical nonhomologous end-joining. Mol Cell. 2014 Sep 18;55(6):829-42.

8. Mao Z, Bozzella M, Seluanov A, Gorbunova V. Comparison of nonhomologous end joining and homologous recombination in human cells. DNA Repair (Amst). 2008 Oct 1;7(10):1765-71.

9. Nordberg A, Minssen T, Holm S, Horst M, Mortensen K, Møller BL. Cutting edges and weaving threads in the gene editing (Я) evolution: reconciling scientific progress with legal, ethical, and social concerns. Journal of Law and the Biosciences. 2018;5(1):35-83.

10. Prakash V, Moore M, Yáñez-Muñoz RJ. Current progress in therapeutic gene editing for monogenic diseases. Molecular Therapy. 2016;24(3):465-74. 11. Paul DS, Soranzo N, Beck S. Functional interpretation of non-coding sequence variation: concepts and challenges. Bioessays. 2014;36(2):191-9.

12. Ma H, Marti-Gutierrez N, Park S-W, Wu J, Lee Y, Suzuki K, et al. Correction of a pathogenic gene mutation in human embryos. Nature. 2017;548(7668):413-9. 
13. Go AS, Mozaffarian D, Roger VL, Benjamin EJ, Berry JD, Borden WB, et al. Heart disease and stroke statistics - 2013 update a report from the American Heart Association. Circulation. 2012:CIR. 0b013e31828124ad.

14. Smith AJ, Deloukas P, Munroe PB. Emerging applications of genomeediting technology to examine functionality of GWAS-associated variants for complex traits. Physiological genomics. 2018.

15. Chadwick AC, Musunuru K. Genome editing for the study of cardiovascular diseases. Current cardiology reports. 2017;19(3):22.

16. Cox DBT, Platt RJ, Zhang F. Therapeutic genome editing: prospects and challenges. Nature medicine. 2015;21(2):121.

17. Limpitikul WB, Dick IE, Tester DJ, Boczek NJ, Limphong P, Yang W, et al. A precision medicine approach to the rescue of function on malignant calmodulinopathic long-QT syndrome. Circulation Research. 2017;120(1):39-48. 18. Larson MH, Gilbert LA, Wang X, Lim WA, Weissman JS, Qi LS. CRISPR interference (CRISPRi) for sequence-specific control of gene expression. Nature protocols. 2013;8(11):2180.

19. Motta BM, Pramstaller PP, Hicks AA, Rossini A. The impact of CRISPR/Cas9 technology on cardiac research: from disease modelling to therapeutic approaches. Stem cells international. 2017;2017.

20. Samulski RJ, Muzyczka N. AAV-mediated gene therapy for research and therapeutic purposes. Annual review of virology. 2014;1:427-51.

21. Kim YG, Cha J, Chandrasegaran S. Hybrid restriction enzymes: zinc finger fusions to Fok I cleavage domain. Proc Natl Acad Sci U S A. 1996 Feb 6;93(3):1156-60.

22. Moscou MJ, Bogdanove AJ. A simple cipher governs DNA recognition by TAL effectors. Science. 2009 Dec 11;326(5959):1501.

23. Wang G, McCain ML, Yang L, He A, Pasqualini FS, Agarwal A, et al. Modeling the mitochondrial cardiomyopathy of Barth syndrome with induced pluripotent stem cell and heart-on-chip technologies. Nature medicine. 2014;20(6):616.

24. Ding Q, Strong A, Patel KM, Ng S-L, Gosis BS, Regan SN, et al. Permanent alteration of PCSK9 with in vivo CRISPR-Cas9 genome editing. Circulation research. 2014;115(5):488-92.

25. Maetzel D, Sarkar S, Wang H, Abi-Mosleh L, Xu P, Cheng AW, et al. Genetic and chemical correction of cholesterol accumulation and impaired autophagy in hepatic and neural cells derived from Niemann-Pick Type $C$ patient-specific iPS cells. Stem cell reports. 2014;2(6):866-80. 
26. Wang Y, Liang P, Lan F, Wu H, Lisowski L, Gu M, et al. Genome editing of isogenic human induced pluripotent stem cells recapitulates long QT phenotype for drug testing. Journal of the American College of Cardiology. 2014;64(5):451-9.

27. Theodoris CV, Li M, White MP, Liu L, He D, Pollard KS, et al. Human disease modeling reveals integrated transcriptional and epigenetic mechanisms of NOTCH1 haploinsufficiency. Cell. 2015;160(6):1072-86.

28. Zhang Z, Ursin R, Mahapatra S, Gallicano GI. CRISPR/CAS9 ablation of individual miRNAs from a miRNA family reveals their individual efficacies for regulating cardiac differentiation. Mechanisms of development. 2018;150:1020.

29. Long C, Li H, Tiburcy M, Rodriguez-Caycedo C, Kyrychenko V, Zhou H, et al. Correction of diverse muscular dystrophy mutations in human engineered heart muscle by single-site genome editing. Science advances. 2018;4(1):eaap9004.

30. Cary LC, Goebel M, Corsaro BG, Wang HG, Rosen E, Fraser MJ. Transposon mutagenesis of baculoviruses: analysis of Trichoplusia ni transposon IFP2 insertions within the FP-locus of nuclear polyhedrosis viruses. Virology. 1989 Sep;172(1):156-69.

31. Ding S, Wu X, Li G, Han M, Zhuang Y, Xu T. Efficient transposition of the piggyBac (PB) transposon in mammalian cells and mice. Cell. 2005 Aug 12;122(3):473-83.

32. Kondrashov A, Duc Hoang M, Smith JG, Bhagwan JR, Duncan G, Mosqueira D, et al. Simplified footprint-free Cas9/CRISPR editing of cardiacassociated genes in human pluripotent stem cells. Stem cells and development. 2018;27(6):391-404.

33. Hai Q, Ritchey B, Robinet P, Alzayed AM, Brubaker G, Zhang J, et al. Quantitative Trait Locus Mapping of Macrophage Cholesterol Metabolism and CRISPR/Cas9 Editing Implicate an ACAT1 Truncation as a Causal Modifier Variant. Arteriosclerosis, thrombosis, and vascular biology. 2018;38(1):83-91. 34. Seeger T, Porteus M, Wu JC. Genome editing in cardiovascular biology. Circulation research. 2017;120(5):778-80.

35. Mendell JR, Rodino-Klapac LR. Duchenne muscular dystrophy: CRISPR/Cas9 treatment. Cell Research. [Review]. 2016;26(5):513-4.

36. Heyer WD, Ehmsen KT, Liu J. Regulation of homologous recombination in eukaryotes. Annual Review of Genetics2010. p. 113-39. 
37. Gramlich M, Pane LS, Zhou Q, Chen Z, Murgia M, Schötterl S, et al. Antisense-mediated exon skipping: A therapeutic strategy for titin-based dilated cardiomyopathy. EMBO Molecular Medicine. [Article]. 2015;7(5):56276.

38. Ehret GB, Ferreira T, Chasman DI, Jackson AU, Schmidt EM, Johnson T, et al. The genetics of blood pressure regulation and its target organs from association studies in 342,415 individuals. Nature Genetics. [Article]. 2016;48(10):1171-84.

39. Nikpay M, Goel A, Won H-H, Hall LM, Willenborg C, Kanoni S, et al. A comprehensive 1000 Genomes-based genome-wide association meta-analysis of coronary artery disease. Nature genetics. 2015;47(10):1121.

40. Port F, Chen H-M, Lee T, Bullock SL. Optimized CRISPR/Cas tools for efficient germline and somatic genome engineering in Drosophila. Proceedings of the National Academy of Sciences. 2014;111(29):E2967-E76.

41. Sebo ZL, Lee HB, Peng Y, Guo Y. A simplified and efficient germlinespecific CRISPR/Cas9 system for Drosophila genomic engineering. Fly. 2014;8(1):52-7.

42. Laflamme MA, Murry CE. Heart regeneration. Nature. 2011;473(7347):326.

43. Torraca V, Sommer F, Kamel S, Otto NA, Meijer AH. CRISPR/Cas9 mutagenesis of zebrafish $\mathrm{Cxcr} 3.3$ suggests opposing functions of atypical and canonical Cxcr3 paralogues on mycobacterial infection control. Exploitation of host chemokine signalling by pathogenic mycobacteria.122.

44. Ablain J, Durand EM, Yang S, Zhou Y, Zon LI. A CRISPR/Cas9 vector system for tissue-specific gene disruption in zebrafish. Developmental cell. 2015;32(6):756-64.

45. Hall B, Limaye A, Kulkarni AB. Overview: generation of gene knockout mice. Current protocols in cell biology. 2009;44(1):19.2. 1-.2. 7.

46. Yang $H$, Wang $H$, Shivalila CS, Cheng AW, Shi L, Jaenisch R. One-step generation of mice carrying reporter and conditional alleles by CRISPR/Casmediated genome engineering. Cell. 2013;154(6):1370-9.

47. Ding Q, Lee $Y-K$, Schaefer EA, Peters DT, Veres A, Kim K, et al. A TALEN genome-editing system for generating human stem cell-based disease models. Cell stem cell. 2013;12(2):238-51.

48. Chadwick AC, Evitt NH, Lv W, Musunuru K. Reduced blood lipid levels with in vivo CRISPR-Cas9 base editing of ANGPTL3. Circulation. 2018;137(9):975-7. 
49. Paton DM. PCSK9 inhibitors: monoclonal antibodies for the treatment of hypercholesterolemia. Drugs Today (Barc). 2016 Mar;52(3):183-92.

50. Brookhouser N, Raman S, Potts C, Brafman DA. May I cut in? Gene editing approaches in human induced pluripotent stem cells. Cells. 2017;6(1):5. 51. Gerlach GF, Wingert RA. Kidney organogenesis in the zebrafish: insights into vertebrate nephrogenesis and regeneration. Wiley Interdisciplinary Reviews: Developmental Biology. 2013;2(5):559-85. 